\title{
Ultraviolet-Visible and Fluorescence Spectroscopy Techniques Are Important Diagnostic Tools during the Progression of Atherosclerosis: Diet Zinc Supplementation Retarded or Delayed Atherosclerosis
}

\author{
Mohamed Anwar K. Abdelhalim, ${ }^{1}$ Sherif A. Abdelmottaleb Moussa, ${ }^{2,3}$ \\ and Yanallah Hussain AL-Mohy ${ }^{1}$ \\ ${ }^{1}$ Department of Physics and Astronomy, College of Science, King Saud University, P.O. Box 2455, Riyadh 11451, Saudi Arabia \\ ${ }^{2}$ Department of Physics, College of Science, Al-Imam Muhammad Ibn Saud Islamic University, P.O. Box 90950, \\ Riyadh 11623, Saudi Arabia \\ ${ }^{3}$ Biochemistry Department, Biophysics group, National Research Centre, Dokki, Giza, Egypt
}

Correspondence should be addressed to Mohamed Anwar K. Abdelhalim; abdelhalimmak@yahoo.com

Received 23 June 2013; Revised 27 August 2013; Accepted 28 August 2013

Academic Editor: Kota V. Ramana

Copyright (C) 2013 Mohamed Anwar K. Abdelhalim et al. This is an open access article distributed under the Creative Commons Attribution License, which permits unrestricted use, distribution, and reproduction in any medium, provided the original work is properly cited.

\begin{abstract}
Background. In this study, we examined whether UV-visible and fluorescence spectroscopy techniques detect the progression of atherosclerosis in serum of rabbits fed on high-cholesterol diet (HCD) and HCD supplemented with zinc (HCD + Zn) compared with the control. Methods. The control rabbits group was fed on $100 \mathrm{~g} /$ day of normal diet. The HCD group was fed on Purina Certified Rabbit Chow supplemented with 1.0\% cholesterol plus $1.0 \%$ olive oil ( $100 \mathrm{~g} /$ day) for the same period. The HCD + Zn group was fed on normal Purina Certified Rabbit Chow plus $1.0 \%$ cholesterol and $1.0 \%$ olive oil supplemented with 470 ppm $\mathrm{Zn}$ for the same feeding period. UV-visible and fluorescence spectroscopy and biochemistry in Rabbit's blood serum and blood hematology were measured in Rabbit's blood. Results. We found that the fluorescent peak of HCD shifted toward UV-visible wavelength compared with the control using fluorescent excitation of serum at $192 \mathrm{~nm}$. In addition, they showed that supplementation of zinc (350 ppm) restored the fluorescent peak closely to the control. By using UV-visible spectroscopy approach, we found that the peak absorbance of HCD (about $280 \mathrm{~nm}$ ) was higher than that of control and that zinc supplementation seemed to decrease the absorbance. Conclusions. This study demonstrates that ultraviolet-visible and fluorescence spectroscopy techniques can be applied as noninvasive techniques on a sample blood serum for diagnosing or detecting the progression of atherosclerosis. The Zn supplementation to rabbits fed on $\mathrm{HCD}$ delays or retards the progression of atherosclerosis. Inducing anemia in rabbits fed on HCD delays the progression of atherosclerosis.
\end{abstract}

\section{Background}

Atherosclerosis is the primary cause of coronary and cardiovascular diseases. Atherosclerosis and heart diseases are major causes of morbidity and mortality in adults in industrialized nations. Atherosclerosis, a stenotic lesion of arterial walls, is a leading cause of death affecting almost one third of humans in developed countries. It can generally be viewed as a form of chronic inflammation that is induced and perturbed by lipid accumulation $[1,2]$. Hyperlipidemia or a high serum triacylglycerol and cholesterol level is considered an important risk factor for the progression of atherosclerosis. The causes of atherosclerosis are mainly due to lifestyle, cholesterol-rich diet, and hereditary [3].

Atherosclerotic lesions are characterized by progressive thickening of the arterial intima layer due to cellular proliferation (foam cells), lipid and cholesterol deposition. Following extracellular lipid accumulation, there is a change in elastin layer and enlargement of upper intima with development of a "collagenous cap" or fibroatheroma plaque. In advanced lesions lipid and necrotic deposits accumulate within the intima, and calcium crystals build-up takes place, leading 
to the ultimate calcification of artery wall. Although many therapies are available for treatment, usually the physician's intervention occurs when the plaque is symptomatic, with high commitment of important organs that need high blood supply, such as heart and brain [4-7].

Fluorescence spectroscopy has been recently explored as a new technique for the diagnosis of disease in human tissue [8-11]. Several groups have demonstrated that normal and atherosclerotic human artery can be differentiated on the basis of their fluorescence emission spectra. Many have suggested that this represents a new technique for determining the histochemical composition of atherosclerotic plaque in vivo and potentially guiding laser angiosurgery catheters [810]. Similarly, it has been shown that neoplastic tissue can be discriminated from nonneoplastic tissue, in a variety of organs, including the colon [10] and lung [12] on the basis of tissue fluorescence emission spectra.

It has been reported that feeding rabbits a high-cholesterol diet resulted in alterations in prooxidant-antioxidant status in several tissues as well as typical atherosclerotic changes in the aorta [13-16].

Fluorescence is the emission of light by a substance following absorption of light or other electromagnetic radiation of a different wavelength. In most cases, the emitted light has a longer wavelength, and therefore lower energy, than the absorbed radiation. However, when the absorbed electromagnetic radiation is intense, it is possible for one molecule to absorb two photons; this two-photon absorption can lead to emission of radiation having a shorter wavelength than the absorbed radiation.

The Fourier-Transform Raman (FT-Raman) spectroscopy is an optical tool which permits less invasive and nondestructive analysis in biological samples with high precision, allowing one to get precision information of biochemical composition from different types of human tissues such as coronary arteries, lung, and colon without tissue removal [1719]. The optical diagnosis has been used in a variety of human diseases, such as near-infrared fluorescence spectroscopy of Alzheimer disease, near-infrared Raman spectroscopy of cervical precancers, and human breast cancer analysis.

Currently there is no noninvasive clinical method available for detecting or diagnosing atherosclerotic plaque content without withdrawing tissue. The ultraviolet-visible and fluorescence spectroscopy techniques have not been well documented in rabbits blood fed on high-cholesterol diet (HCD) and HCD supplemented with high zinc ( $\mathrm{Zn}$ ) level. Thus, the aim of this study is to diagnosis or detect the progression of atherosclerosis as well as the delay or retard of the progression of atherosclerosis applying ultravioletvisible and fluorescence spectroscopy techniques on rabbit's blood sample. For this reason, to diagnose atherosclerosis with noninvasive techniques, a group of rabbits are fed on HCD for a feeding period of 12 weeks, and rabbits are fed on $\mathrm{HCD}$ supplemented with high $\mathrm{Zn}$ level for the same feeding period of time; then ultraviolet-visible and fluorescence spectroscopy techniques will be applied as noninvasive techniques on rabbit's blood sample.

\section{Materials and Methods}

2.1. Animals. The atherosclerotic model used in this study was the New Zealand white rabbit (male, 12 weeks old), obtained from the Laboratory Animal Center (College of Pharmacy, King Saud University). Fifteen rabbits were individually caged and divided into the following. The 1st group is control group $(n=8)$, the 2nd group is high-cholesterol diet (HCD; $n=8$ ) group, and the 3rd group is the HCD group supplemented with high $\mathrm{Zn}$ level $(n=8)$. The control group $(n=5)$ was fed on $100 \mathrm{~g} /$ day of NOR diet (Purina Certified Rabbit Chow no. 5321; Research Diet Inc., New Jersey, USA) for a feeding period of 12 weeks. Chemical composition of the laboratory NOR rabbit diet (Purina Certified Rabbit Chow \# 5321) [4-6]. The HCD group $(n=5)$ was fed on NOR Purina Certified Rabbit Chow no. 5321 supplemented with 1.0\% cholesterol plus $1.0 \%$ olive oil (100 g/day) for the same feeding period of time. The HCD + Zn group was fed on NOR Purina Certified Rabbit Chow plus 1.0\% cholesterol and 1.0\% olive oil supplemented with $350 \mathrm{ppm} \mathrm{Zn} \mathrm{(total} \mathrm{estimate} 470 \mathrm{ppm}$ $\mathrm{Zn}$ ) for the same feeding period of time. The animals were sacrificed by intravenous injection of Hypnorm $(0.3 \mathrm{~mL} / \mathrm{kg})$ in accordance with the guidelines approved by King Saud University Local Animal Care and Use Committee.

2.2. Collection of Blood Serum. Blood samples were collected from control, HCD, and $\mathrm{HCD}+\mathrm{Zn}$ groups of animals, following an overnight fast, and collectively analyzed. Blood samples of $2 \mathrm{~mL}$ were obtained from the rabbits via vein puncture of an antecubital vein. The blood was collected into two polypropylene tubes, one for serum and one for plasma. The blood for plasma was collected in heparin. The serum was prepared by allowing the blood to clot at $37^{\circ} \mathrm{C}$ and centrifuge at $3000 \mathrm{rpm}$ for $10 \mathrm{~min}$.

\subsection{UV-Visible Spectroscopy}

2.3.1. Absorbance Spectra. Rabbit blood serum absorbance of control, $\mathrm{HCD}$, and $\mathrm{HCD}+\mathrm{Zn}$ groups was measured using a UV-VIS double beam spectrophotometer (UV-1601 PC, Shimadzu, Japan; H14 grating UV) through optical resolution of $0.4 \mathrm{~nm}$. Absorbance measurements were obtained over wavelength range of $200-800 \mathrm{~nm}$ at room temperature using quartz cuvettes $(1 \mathrm{~cm}$ path length). The cuvettes were cleaned before each use by sonicating for $5 \mathrm{~min}$ in deionized water followed by rinsing with deionized water. The $\mathrm{pH}$ for different blood serum samples was kept constant during the measurements.

2.3.2. Fluorescence Spectroscopy. Fluorescence measurements were performed in rabbit's blood serum samples using a FluoroMax-2 JOBAN YVON-SPEX, Instruments S.A., Inc., France. The fluorescence measurements were made over wavelength range of $190-400 \mathrm{~nm}$ and at 2 different excitation values (192 and $278 \mathrm{~nm}$ ) on series of samples with a $10 \mathrm{~mm}$ light path cuvette.

2.4. Biochemical and Hematological Investigations. Blood samples $(2 \mathrm{~mL})$ were obtained from the rabbits via vein 


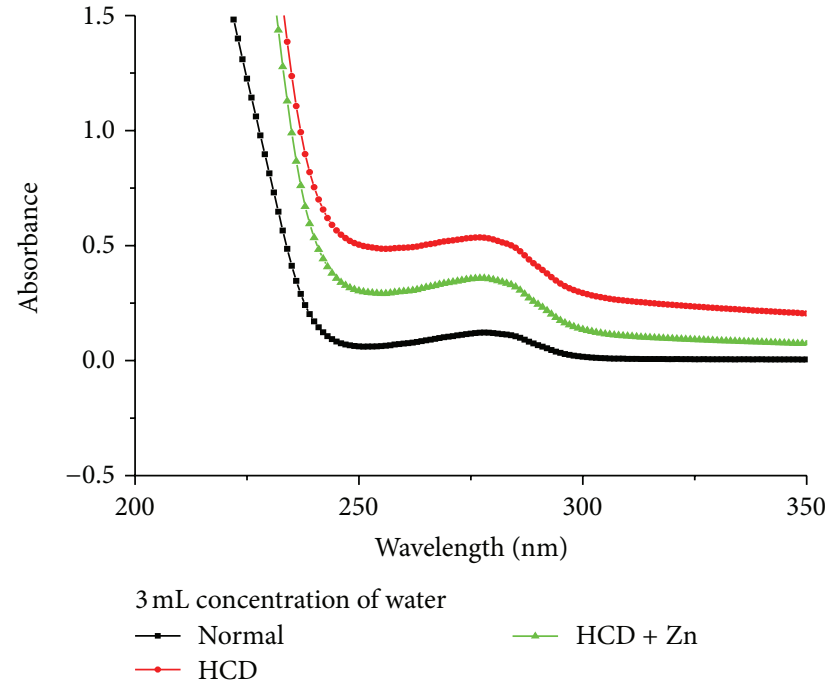

FIGURE 1: It shows absorbance against wavelength $(\mathrm{nm})$ for a sample of rabbit's blood serum taken from control, $\mathrm{HCD}$, and $\mathrm{HCD}+\mathrm{Zn}$ groups ( $3 \mathrm{~mL}$ concentration of water).

puncture of an antecubital vein. Blood was collected into two polypropylene tubes, namely, one for serum and one for plasma. The blood for plasma was collected in heparin. Serum was prepared by allowing the blood to clot at $37^{\circ} \mathrm{C}$ and centrifugation at $3000 \mathrm{rpm}$ for ten minutes. A hematological autoanalyzer (Orphee Mythic 22 Hematological Analyzer, Diamond Diagnostic, USA) was used to determine different hematological and dimensional parameters, such as red blood cells (RBC), white blood cells (WBCs), hemoglobin (HB), hematocrit (HCT), mean corpuscular volume $(\mathrm{MCV})$, mean corpuscular hemoglobin $(\mathrm{MCH})$, mean corpuscular hemoglobin concentration (MCHC), red blood cell distribution width (RDW), neutrophils\%, lymphocytes\%, monocytes $\%$, eosinophils\%, basophils $\%$, mean platelet volume (MPV), platelet distribution width (PDW)\%, plateletcrit (PCT)\%, and platelets (PLTs). Serum TC and TG levels were analyzed by the enzymatic method used in the clinical laboratory center of King Khaled Hospital. HDL concentration was determined by the previously reported method (Lee et al., 1998).

2.5. Histopathological Analysis. Animals were weighted and examined daily for health and behavioral changes following $\mathrm{HCD}$ and $\mathrm{HCD}+\mathrm{Zn}$ administration. To evaluate the morphological changes, several samples from different rabbit organs (liver, heart, lung, kidney, and aorta) were cut rapidly, fixed in neutral buffered formalin (10\%), and dehydrated through a series of ethanol grades $(70,80,90,95$, and $100 \%)$. The rabbit samples were then cleared in 2 changes of xylene, impregnated with 2 changes of molten paraffin wax, embedded, and blocked. Paraffin sections (4-5 um) were stained with hematoxylin and eosin according to [20].

Traditional histopathological technique was performed. Hematoxylin and eosin, Verhoeff, Masson, and Picrosirius staining were used for tissue structure observation. The

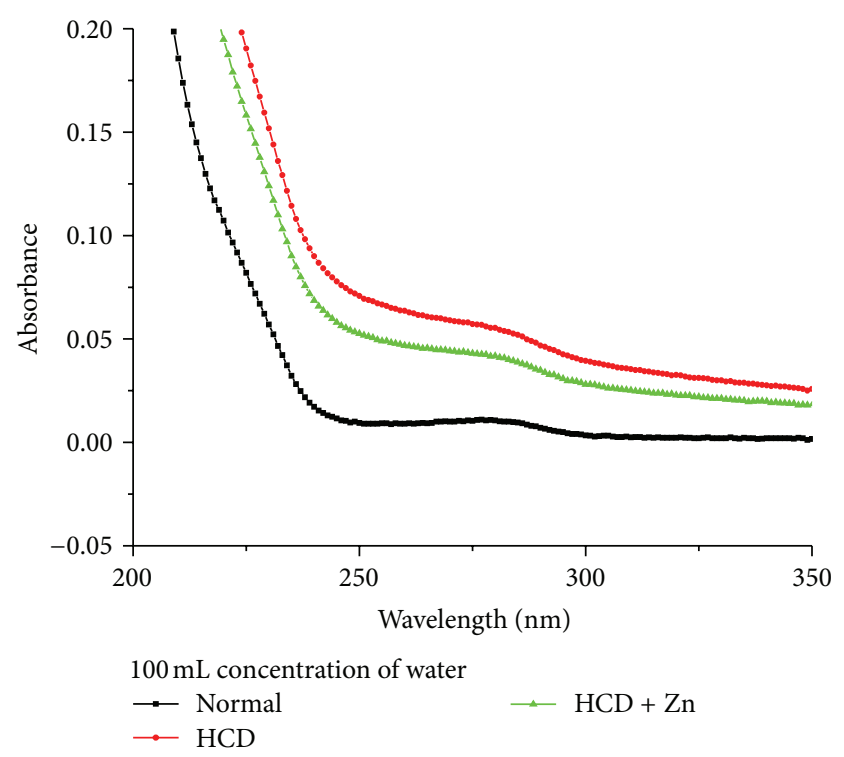

FIGURE 2: It shows absorbance against wavelength $(\mathrm{nm})$ for a sample of rabbit's blood serum taken from control, HCD, and HCD $+\mathrm{Zn}$ groups ( $100 \mathrm{~mL}$ concentration of water).

histopathological classification was made by a board certified pathologist.

2.6. Statistical Analysis. We expressed the results for UVVisible and fluorescence spectroscopy, blood biochemistry, and hematology as mean \pm standard error (Mean \pm SE). To assess the significance of the differences between control, $\mathrm{HCD}$, and $\mathrm{HCD}+\mathrm{Zn}$, we performed the statistical analysis using one-way analysis of variance (ANOVA) for repeated measurements, with significance assessed at $5 \%$ confidence level.

\section{Results and Discussions}

To diagnose or detect and delay the progression of atherosclerosis, noninvasive techniques such as ultraviolet-visible and fluorescence spectroscopy techniques were applied on rabbit's blood sample fed on HCD and HCD supplemented with high $\mathrm{Zn}$ level for a feeding period of 12 weeks.

3.1. UV-Visible Spectroscopy of Rabbit's Blood Serum. The normal blood is characterized by Five hemoglobin $(\mathrm{Hb})$ peaks: the peak at $280 \mathrm{~nm}$ corresponds to the aromatic amino acids, at $340 \mathrm{~nm}$ corresponds to globin-heme interaction, at $420 \mathrm{~nm}$ corresponds to the heme, the peak at $540 \mathrm{~nm}$ corresponds to heme-heme interactions, and at $578 \mathrm{~nm}$ corresponds to hemoglobin oxygen affinity $[7,21]$.

Figures 1 and 2 show the average absorbance against wavelength $(\mathrm{nm})$ for rabbit's blood serum taken from control, $\mathrm{HCD}$, and $\mathrm{HCD}+\mathrm{Zn}$ groups $(N=5)$. In each group, the absorbance peak for rabbits fed on HCD significantly increased compared with the control, while it returned towards the control absorbance peak for rabbits fed on 


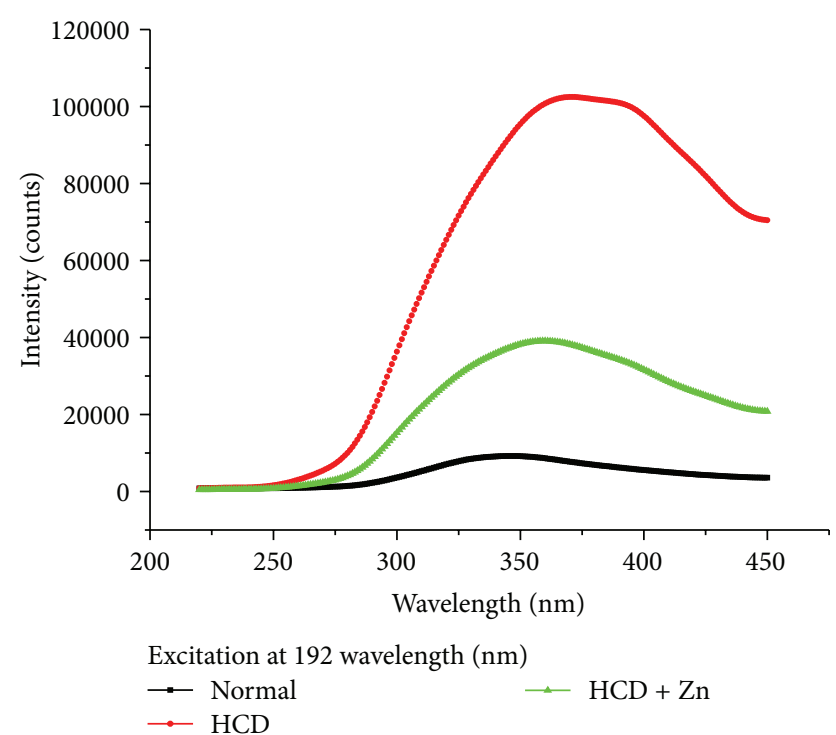

FIGURE 3: Fluorescence intensity (counts) against wavelength (nm) for a sample of rabbit's blood serum taken from control, HCD, and $\mathrm{HCD}+\mathrm{Zn}$ groups at excitation wavelength of $192 \mathrm{~nm}$.

$\mathrm{HCD}+\mathrm{Zn}$. The absorbance peak for control was significantly different from those for HCD and HCD $+Z n$.

3.2. Fluorescence Spectroscopy of Rabbit's Blood Serum. We have observed two peaks at wavelengths $195 \mathrm{~nm}$ and $280 \mathrm{~nm}$ using the UV-Visible spectroscopy (Figures 1 and 2). Thus in the methodological design of this study we have done excitation at wavelengths 192 and $278 \mathrm{~nm}$ for fluorescence spectroscopic analysis of rabbit blood serum [7]. Figure 3 shows fluorescence intensity (counts) against wavelength $(\mathrm{nm})$ for a sample of rabbit's blood serum taken from control, HCD, and $\mathrm{HCD}+\mathrm{Zn}$ groups. In which an excitation wavelength of $192 \mathrm{~nm}$ was used to induce autofluorescence in a sample rabbit's blood during the progression of atherosclerosis.

Figure 3 shows that the maximum fluorescence peak wavelength shifted towards the visible region for HCD group, and it returned towards the control maximum fluorescence peak wavelength for rabbits fed on $\mathrm{HCD}+\mathrm{Zn}$. The fluorescence emission from normal rabbit blood serum was significantly different from those for $\mathrm{HCD}$ and $\mathrm{HCD}+\mathrm{Zn}$.

One of the first attempts to use laser spectroscopy for plaque diagnosis was made by Kittrell [12], in which an excitation wavelength of $480 \mathrm{~nm}$ was used to induce autofluorescence in aorta affected with atherosclerosis. They demonstrated that fluorescence emission from normal artery was significantly different from fibrous plaque. The research was focused on autofluorescence emission for tissue characterization and for use as laser angioplasty guidance system. However, due to the poor biochemical information carried out by fluorescence emission spectrum, the use of Raman spectroscopy for biological tissue classification has been evidenced [17-19].

Figure 4 shows fluorescence intensity (counts) against wavelength $(\mathrm{nm})$ for a sample of rabbit's blood serum taken

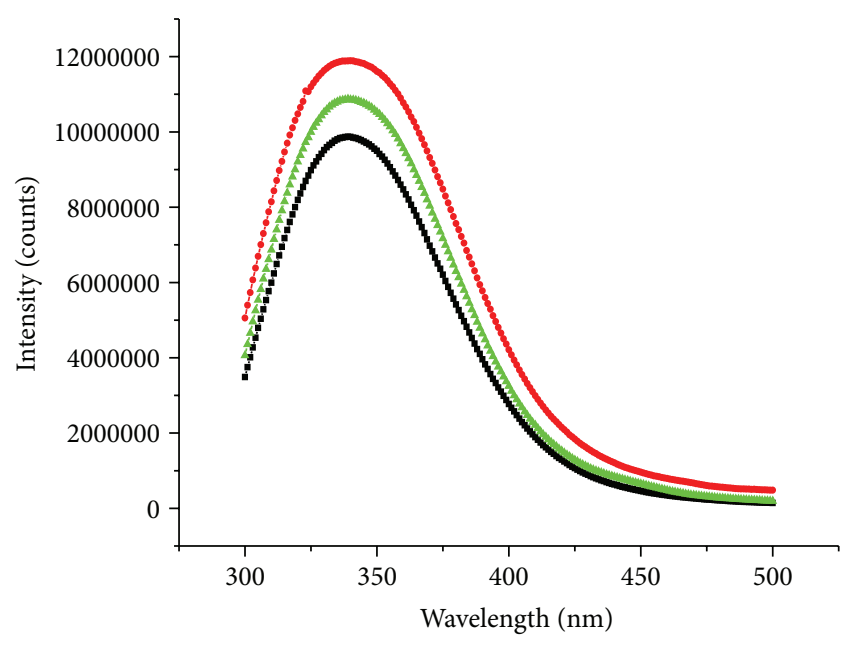

Excitation at wavelength $278(\mathrm{~nm})$

$\rightarrow$ Normal
$\rightarrow \mathrm{HCD}$

FIGURE 4: Fluorescence intensity (counts) against wavelength (nm) for a sample of rabbit's blood serum taken from control, HCD, and $\mathrm{HCD}+\mathrm{Zn}$ groups at excitation wavelength of $278 \mathrm{~nm}$.

from control, $\mathrm{HCD}$, and $\mathrm{HCD}+\mathrm{Zn}$ groups. In which an excitation wavelength of $278 \mathrm{~nm}$ is used to induce autofluorescence in a sample rabbit's blood during the progression of atherosclerosis. The maximum fluorescence peak wavelength returned towards the control maximum fluorescence peak wavelength for rabbits fed on $\mathrm{HCD}+\mathrm{Zn}$. The fluorescence emission from normal rabbit blood serum was significantly different from those for HCD and HCD $+\mathrm{Zn}$.

In this study, we have observed the same results in each group $(N=5)$, so we have provided the average fluorescent peak value of each group $(N=5)$ in terms of mean $\pm S D$, and the statistical analysis between control, HCD, and HCD $+\mathrm{Zn}$ was statistically significant.

The best spectroscopic results were obtained with an excitation wavelength of $325 \mathrm{~nm}$. In samples with severe atherosclerotic lesions, the fluorescence spectra showed a significant reduction of the emitted wavelength intensities when compared to normal tissue. There was a clear separation of the fluorescence spectra between normal and mild as well as between normal and severe atherosclerotic lesions; normal tissue showed an increased intensity in the range from $420 \mathrm{~nm}$ to $540 \mathrm{~nm}$, whereas atherosclerotic lesions had no or only a small peak at $480 \mathrm{~nm}$.

Silveira et al. [22] have taken comparable result, where 111 fragments of coronary arteries were analyzed by rearinfrared Raman spectroscopy using 95 samples correctly classified, with $87 \%$ agreement and high sensitivity and specificity values. It has shown that FT-Raman can accurately discriminate biochemical differences between normal and atherosclerotic plaque and can be used to spectrally classify them. These results suggest that FT-Raman spectroscopy may be turned to a promising diagnostic technique in therapeutics such as laser angioplasty and as a Raman guidance system. FT-Raman spectrum not only discriminates between healthy 


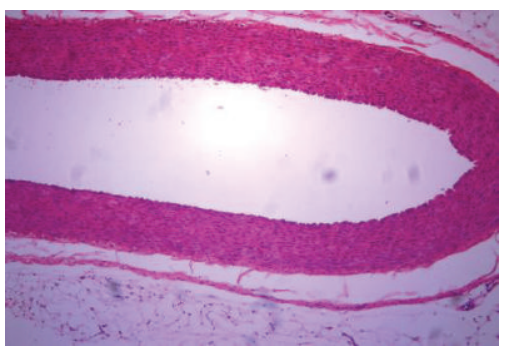

$\times 400$

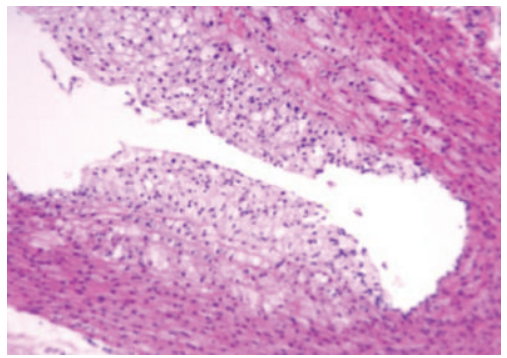

$\times 400$

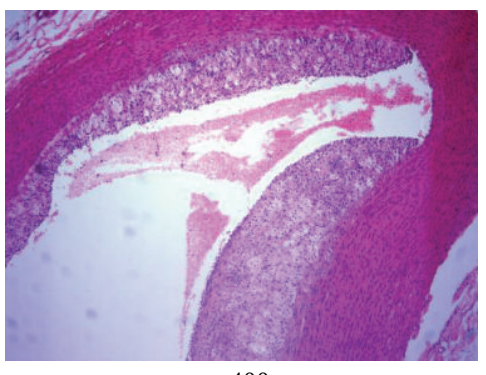

$\times 400$

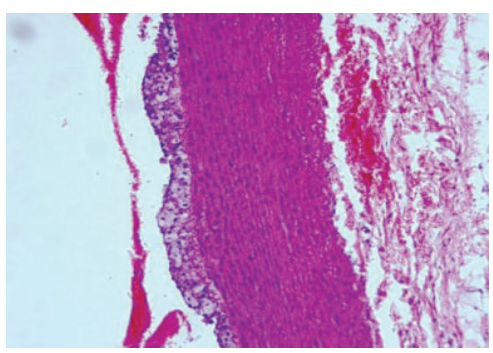

(a)

(b)
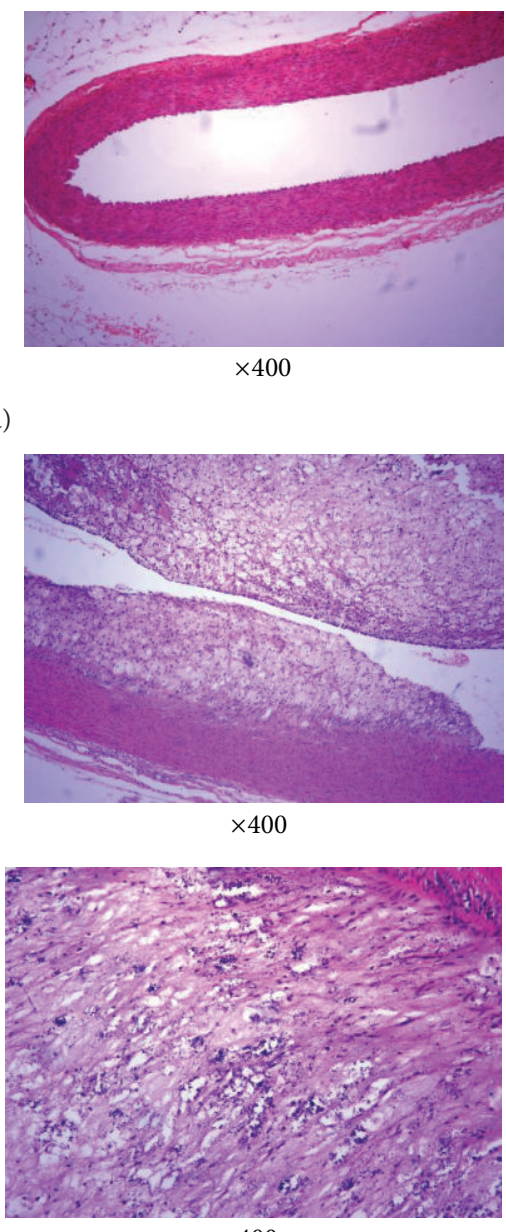

$\times 400$

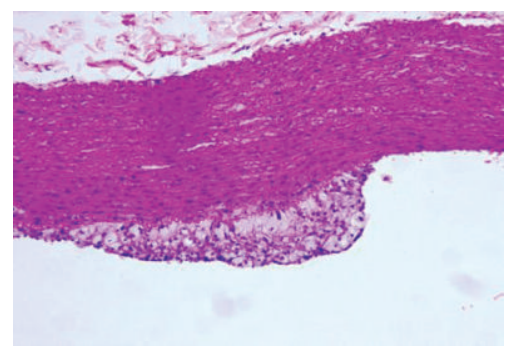

(c)

Figure 5: (a) Shows normal aorta. (b) Shows high-density cholesterol aorta. (c) Shows high-density cholesterol + zinc aorta.

and pathological artery wall with molecular specificity, but also provides exclusive diagnostic signature for each tissue. This technique will also enable the study of atherogenesis in situ, allowing the investigation of the disease progression as well as the response of different therapeutic modalities.

Proteins are dynamic and essential to their function. A decrease in absorbance at $280 \mathrm{~nm}$ indicates abnormal motion and reflects a deviation from normal structure and function. The extent of deviation reflects the degree of globin unfolding and random motion of $\mathrm{Hb}$ molecule under different levels of oxidative stress [23]. Hypercholesterolemia can increase the cholesterol content of platelets, polymorphonuclear leukocytes, and endothelial cells so that endothelial and smooth muscle cells, neutrophils, and platelets may be sources of oxygen-free radicals [24]. It becomes evident that oxidative stress occurs when there is an excessive production of free radicals in the face of defective antioxidant defenses.

3.3. Histological Analysis of Rabbit's Aorta. Figure 5 ((a) Normal Aorta) demonstrates dilated, benign-looking aorta. Aortic section of rabbit showed well-formed dilated lumen 
TABLE 1: Concentrations of lipids and lipoproteins in control, HCD, and HCD + Zn Rabbits $\left({ }^{*}\right.$ means $\left.P<0.05\right)$.

\begin{tabular}{lccc}
\hline Chemical parameters (mg/dL) & Control $(n=5)$ & HCD $(n=5)$ & HCD + Zn $(n=5)$ \\
\hline Total cholesterol (TC) & $65.2 \pm 8.71$ & $658.95 \pm 95.27^{*}$ & $93.12 \pm 5.5^{*}$ \\
Low-density lipoprotein (LDL) & $43.6 \pm 7.41$ & $677.2 \pm 11.1^{*}$ & $95 \pm 24.1^{*}$ \\
High-density lipoprotein (HDL) & $12.37 \pm 1.65$ & $17.32 \pm 1.55$ & $13 \pm 1.6$ \\
Triglyceride & $89.24 \pm 9.71$ & $197.25 \pm 32.25^{*}$ & $109 \pm 10.3^{*}$ \\
\hline
\end{tabular}

TABLE 2: Complete blood picture of control, HCD, and HCD + Zn rabbits $\left({ }^{*}\right.$ means $\left.P<0.05\right)$.

\begin{tabular}{lccc}
\hline Blood index & $\begin{array}{c}\text { Control } \\
(n=5)\end{array}$ & HCD $(n=5)$ & $\begin{array}{c}\text { HCD }+\mathrm{Zn} \\
(n=5)\end{array}$ \\
\hline WBC (K/UL) count & $6.95 \pm 0.44$ & $13.9 \pm 0.57$ & $8 \pm 0.52$ \\
LYM\% & $43.73 \pm 2.02$ & $60 \pm 2.00^{*}$ & $50 \pm 2.00$ \\
MON\% & $2.23 \pm 1.27$ & $1.15 \pm 0.15^{*}$ & $2.26 \pm 0.97$ \\
NEU\% & $46.55 \pm 2.69$ & $35.7 \pm 2.10^{*}$ & $43.21 \pm 2.33$ \\
EOS\% & $2.0 \pm 0.57$ & $1.33 \pm 0.66^{*}$ & $1.6 \pm 0.50$ \\
BAS\% & $0.50 \pm 0.18$ & $0.45 \pm 0.05$ & $1 \pm 0.13$ \\
RBCs (K/UL) count & $6.10 \pm 0.14$ & $4.23 \pm 0.48^{*}$ & $5.64 \pm 0.50$ \\
HGB (g/dL) & $12.53 \pm 0.28$ & $10.56 \pm 0.31^{*}$ & $12.47 \pm 0.83$ \\
HCT\% & $40.41 \pm 0.81$ & $32.42 \pm 2.05^{*}$ & $34.34 \pm 5.19$ \\
MCV & $66.27 \pm 0.77$ & $79.62 \pm 6.75^{*}$ & $70.56 \pm 6.20$ \\
MCH & $20.43 \pm 0.40$ & $26.8 \pm 4.14$ & $28.62 \pm 6.36$ \\
MCHC & $31.01 \pm 0.15$ & $32.92 \pm 1.98$ & $33.98 \pm 2.56$ \\
RDW & $13.2 \pm 0.34$ & $16.36 \pm 1.09^{*}$ & $14.42 \pm 1.87$ \\
PLT & $398.83 \pm 50.00$ & $328.87 \pm 52^{*}$ & $125.35 \pm 12.45$ \\
MPV & $6.6 \pm 0.21$ & $8.7 \pm 0.44^{*}$ & $6.1 \pm 0.29$ \\
PCT & $0.25 \pm 0.03$ & $0.35 \pm 0.06^{*}$ & $0.22 \pm 0.05$ \\
PDW & $9.97 \pm 0.55$ & $11.1 \pm 0.76^{*}$ & $9.23 \pm 0.82$ \\
\hline
\end{tabular}

lined by endothelial cells surrounded by their muscular wall. No pathological feature was detected.

Figure 5 ((b) High-density cholesterol Aorta) demonstrates aortic sections of rabbit aorta and showed dilated lumen with different grades of vascular occlusion of the lumen by arthrosclerosis with subintimal deposition of fibrofatty tissue associated with smooth muscle proliferation ranging from partial to subtotal closure of lumen with occasional foci of calcifications.

Figure 5 ((c) high-density cholesterol + Zinc aorta) demonstrates sections of aorta rabbits and showed variable degree of elevation and partial closure of aortic lumen due to atherosclerosis with some differentiation between aortic sections with HCD.

3.4. Biochemical Analysis of Rabbit's Blood Serum. Table 1 shows the levels of TC, TG, and HDL concentrations in control, $\mathrm{HCD}$, and $\mathrm{HCD}+\mathrm{Zn}$ rabbits. Table 1 indicates significant increases in TC, TG, and HDL in HCD and HCD $+\mathrm{Zn}$ compared with control, while significant decreases in TC, TG, and HDL in HCD + Zn compared with HCD.

Table 2 shows that RBCs count, hemoglobin, HCT\%, monocyte $\%$, neutrophils $\%$, eosinophils $\%$, and basophiles $\%$, decreased in HCD compared with the control, while WBCs count, LYM\%, MCV, MCH, MCHC, RDW, PLT, MPV, PCT, and RDW increased in HCD compared with control. The zinc supplementation to HCD improved all blood indices during the progression of atherosclerosis. The deficiency in RBCs, $\mathrm{HGB}$, and $\mathrm{HCT} \%$ are very important factors during the progression of atherosclerosis. The dimensional parameters such as RDW, MPV, PCT, and PDW, and PLT count can also be used as predictor factors for atherosclerosis and cardiovascular diseases.

This study suggests that further experiments will be performed taking into consideration lipid peroxidation assay (MDA or TBARS) to show the degree of oxidation in rabbit serum, which will support and link the UV-Visible, fluorescence spectroscopy data, and histopathological data in each group. In addition, we will examine the degree of atherosclerosis in the aorta by measuring the intimal and media thickness of the aorta and expressing it in terms of intimal/media ratio. Finally, we will perform UV-Visible and fluorescence spectroscopy of rabbit serum at different time points (for example 4, 8, and 12 weeks) instead of only one time point (12 weeks), after feeding with HCD to monitor whether the UV-Vis/fluorescent spectroscopy data correlate with the progression of atherosclerosis.

\section{Conclusions}

To diagnose or detect and delay the progression of atherosclerosis, noninvasive techniques such as ultraviolet-visible and fluorescence spectroscopy techniques were applied on blood sample of rabbits fed on HCD and HCD supplemented with high $\mathrm{Zn}$ level for a feeding period of 12 weeks.

Hypercholesterolemia can increase the cholesterol content of platelets, polymorphonuclear leukocytes, and endothelial cells so that endothelial and smooth muscle cells, neutrophils and platelets may be sources of oxygen-free radicals. It becomes evident that oxidative stress occurs when there is an excessive production of free radicals in the face of defective antioxidant defenses.

At excitation of $192 \mathrm{~nm}$, the fluorescence peak shifted towards the visible region for HCD group, while it returned towards the control fluorescence peak for rabbits fed on $\mathrm{HCD}+\mathrm{Zn}$. The fluorescence emission from normal rabbit blood serum was significantly different from those for HCD and $\mathrm{HCD}+\mathrm{Zn}$.

At excitation of $278 \mathrm{~nm}$, the fluorescence peak for normal rabbit blood serum was significantly different from those for $\mathrm{HCD}$ and $\mathrm{HCD}+\mathrm{Zn}$.

The absorbance peak for rabbits fed on HCD significantly increased compared with the control, while it returned towards the control absorbance peak for rabbits fed on 
$\mathrm{HCD}+\mathrm{Zn}$. The absorbance peak for control was significantly different from those for HCD and $\mathrm{HCD}+\mathrm{Zn}$.

Proteins are dynamic and essential to their function. An increase in absorbance at $280 \mathrm{~nm}$ might indicate abnormal protein motion and reflects a deviation from the normal structure and function. The extent of deviation reflects the degree of globin unfolding and random motion of $\mathrm{Hb}$ molecule under different levels of oxidative stress.

This study demonstrates that ultraviolet-visible and fluorescence spectroscopy techniques can be applied as noninvasive techniques on a sample blood serum for diagnosing or detecting the progression of atherosclerosis. The $\mathrm{Zn}$ supplementation to rabbits fed on HCD delays or retards the progression of atherosclerosis. Inducing anemia in rabbits fed on HCD delays the progression of atherosclerosis. These conclusions might be supported by further histological investigations in rabbit's different organs.

\section{Conflict of Interests}

The authors declare that they have no conflict of interests.

\section{Authors' Contribution}

Mohamed Anwar K. Abdelhalim, Sherif A. Abdelmottaleb Moussa, and Yanallah Hussain AL-Mohy have analyzed data. Mohamed Anwar K. Abdelhalim and Sherif A. Abdelmottaleb Moussa have interpreted and written the final draft of this paper. The animal model used in this study was obtained from the Laboratory Animal Center (College of Pharmacy, King Saud University, Saudi Arabia). Mohamed Anwar K. Abdelhalim has conceived the study and its design and obtained research grants for this study. Moreover, both authors have read and approved the final paper.

\section{Acknowledgment}

The authors would like to extend their sincere appreciation to the Deanship of Scientific Research at King Saud University for the funding of this research through the research Group Project no. RGP-VPP-285.

\section{References}

[1] C. K. Glass and J. L. Witztum, "Atherosclerosis: the road ahead," Cell, vol. 104, no. 4, pp. 503-516, 2001.

[2] R. Ross, "Atherosclerosis-an inflammatory disease," The New England Journal of Medicine, vol. 340, no. 2, pp. 115-126, 1999.

[3] R. S. Cotran, V. Kumar, and T. Collins, Patologia Estrutural E Funcional, Guanabara Koogan, Rio de Janeiro, Brazil, 2000.

[4] M. A. K. Abdelhalim and M. Mady, "Liver uptake of gold nanoparticles after intraperitoneal administration in vivo: a fluorescence study," Lipids in Health and Disease, vol. 10, article 195, 2011.

[5] M. Abdelhalim and B. M. Jarrar, "Gold nanoparticles administration induced prominent inflammatory, central vein intima disruption, fatty change and Kupffer cells hyperplasia," Lipids in Health and Disease, vol. 10, article 133, 2011.
[6] M. A. K. Abdelhalim and B. M. Jarrar, "The appearance of renal cells cytoplasmic degeneration and nuclear destruction might be an indication of GNPs toxicity," Lipids in Health and Disease, vol. 10, article 147, 2011.

[7] M. A. K. Abdelhalim, "The bioaccumulation and toxicity induced by gold nanoparticles in rats in vivo can be detected by ultraviolet-visible (UV-visible) spectroscopy," African Journal of Biotechnology, vol. 11, no. 39, pp. 9399-9406, 2012.

[8] E. Braunwald, Tratado de Medicina Cardiovascular, Editora Roca LTDA, São Paulo, Brazil, 1999.

[9] R. S. Cotran, V. Kumar, and C. T. Robbins, Patología Estrutural e Funcional, Guanabara Koogan, Rio de Janeiro, Brazil, 2000.

[10] H. C. Stary, "Evolution and progression of atherosclerotic lesions in coronary arteries of children and young adults," Atherosclerosis Supplements, vol. 9, no. 1, pp. I19-I32, 1989.

[11] M. Keijzer, R. Richards-Kortum, S. L. Jacques, and M. S. Feld, "Fluorescence spectroscopy of turbid media: autofluorescence of human aorta," Applied Optics, vol. 28, no. 20, pp. 4286-4292, 1989.

[12] C. Kittrell, R. L. Willett, and C. De Los Santos-Pacheo, "Diagnosis of fibrous arterial atherosclerosis using fluorescence," Applied Optics, vol. 24, no. 15, pp. 2280-2281, 1985.

[13] A. Warnholtz, H. Mollnau, M. Oelze, M. Wendt, and T. Münzel, "Antioxidants and endothelial dysfunction in hyperlipidemia," Current Hypertension Reports, vol. 3, no. 1, pp. 53-60, 2001.

[14] A. J. Lusis, “Atherosclerosis," Nature, vol. 407, no. 6801, pp. $233-$ 241, 2000.

[15] R. Kohen and A. Nyska, "Oxidation of biological systems: oxidative stress phenomena, antioxidants, redox reactions, and methods for their quantification," Toxicologic Pathology, vol. 30, no. 6, pp. 620-650, 2002.

[16] M. A. K. Abdelhalim and S. A. Moussa, "Biochemical changes of hemoglobin and osmotic fragility of red blood cells in high fat diet rabbits," Pakistan Journal of Biological Sciences, vol. 13, no. 2, pp. 73-77, 2010.

[17] P. Weinmann, M. Jouan, N. Quy Dao et al., "Quantitative analysis of cholesterol and cholesteryl esters in human atherosclerotic plaques using near-infrared Raman spectroscopy," Atherosclerosis, vol. 140, no. 1, pp. 81-88, 1998.

[18] T. J. Römer, J. F. Brennan III, M. Fitzmaurice et al., "Histopathology of human coronary atherosclerosis by quantifying its chemical composition with Raman spectroscopy," Circulation, vol. 97, no. 9, pp. 878-885, 1998.

[19] G. Deinum, D. Rodriguez, T. J. Römer, M. Fitzmaurice, J. R. Kramer, and M. S. Feld, "Histological classification of Raman spectra of human coronary artery atherosclerosis using principal component analysis," Applied Spectroscopy, vol. 53, no. 8, pp. 938-942, 1999.

[20] A. E. Pearse, Histochemistry. Theoritical and Applied. Analytical Technology, vol. 2, Churchill-Livingstone, Edinburgh, UK, 4th edition, 1985.

[21] J. V. Dacie and S. M. Lewis, Practical Haematology, Churchill Livingstone, Edinburgh, UK, 7th edition, 1991.

[22] L. Silveira Jr., S. Sathaiah, R. A. Zngaro, M. T. T. Pacheco, M. C. Chavantes, and C. A. G. Pasqualucci, "Correlation between near-infrared Raman spectroscopy and the histopathological analysis of atherosclerosis in human coronary arteries," Lasers in Surgery and Medicine, vol. 30, no. 4, pp. 290-297, 2002.

[23] A. Jetsrisuparb, K. Sanchaisuriya, G. Fucharoen et al., "Development of severe anemia during fever episodes in patients with hemoglobin E trait and hemoglobin $\mathrm{H}$ disease combinations," 
Journal of Pediatric Hematology/Oncology, vol. 28, no. 4, pp. 249-253, 2006.

[24] H. Esterbauer, J. Gebicki, H. Puhl, and G. Jurgens, "The role of lipid peroxidation and antioxidants in oxidative modification of LDL," Free Radical Biology and Medicine, vol. 13, no. 4, pp. 341390, 1992. 

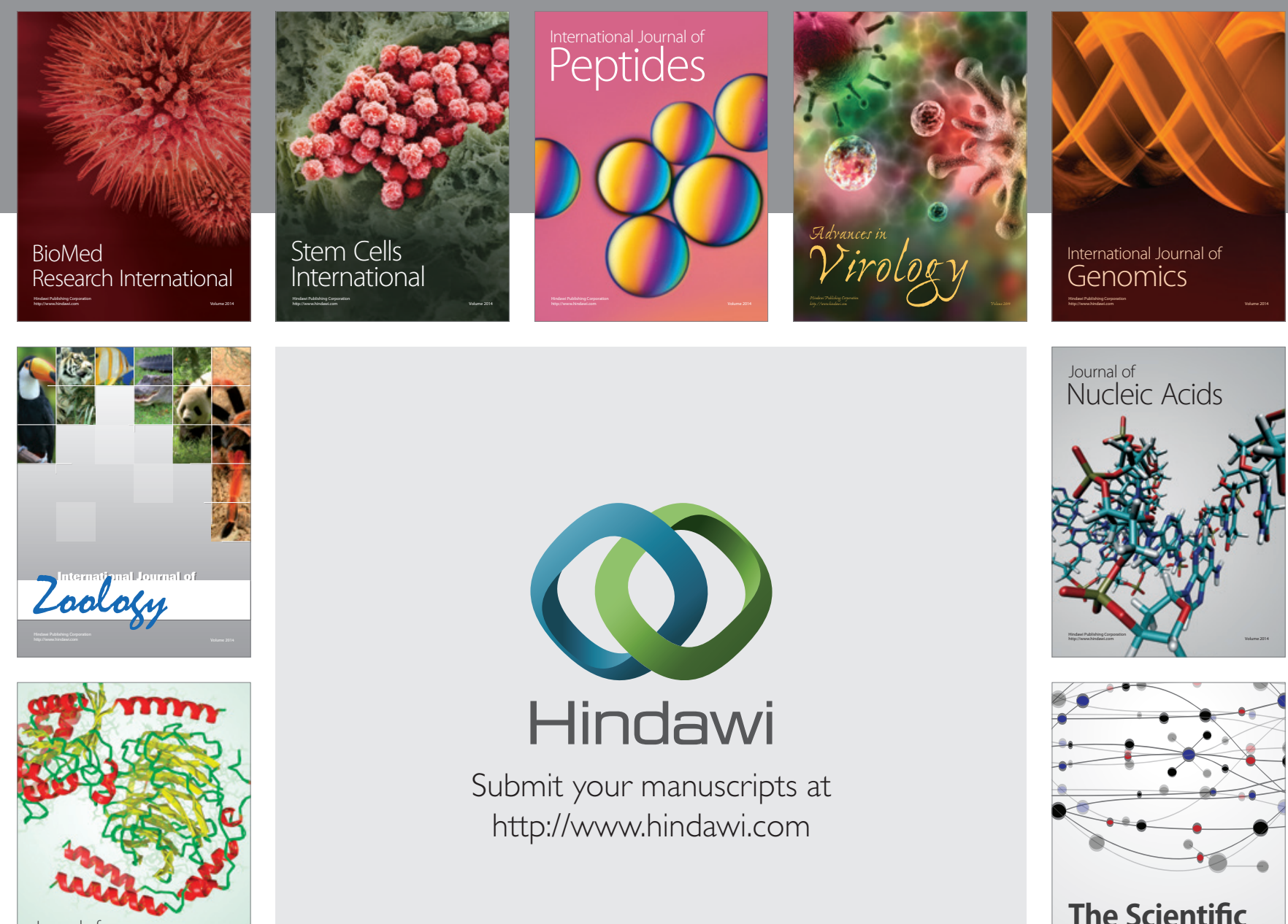

Submit your manuscripts at

http://www.hindawi.com

Journal of
Signal Transduction
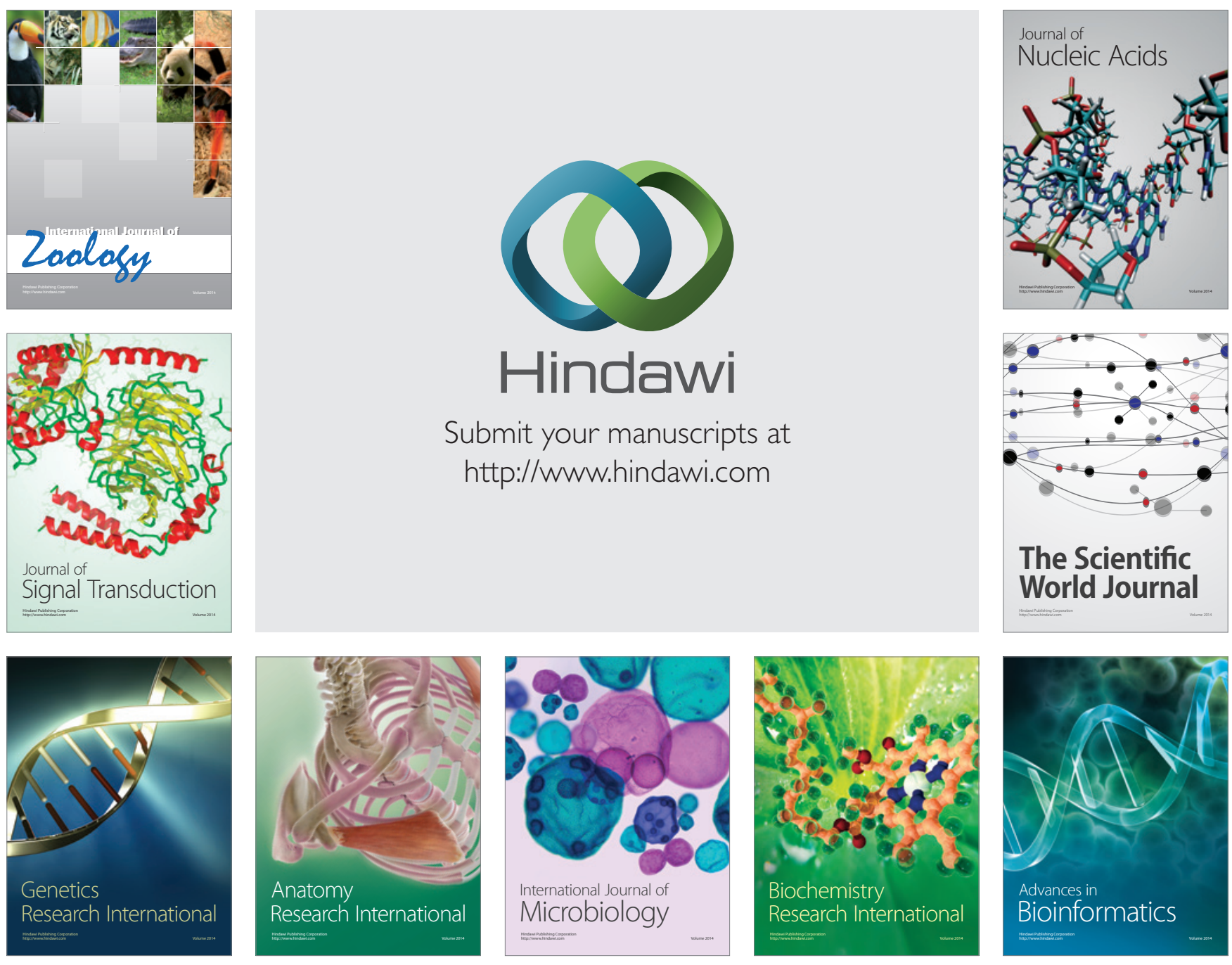

The Scientific World Journal
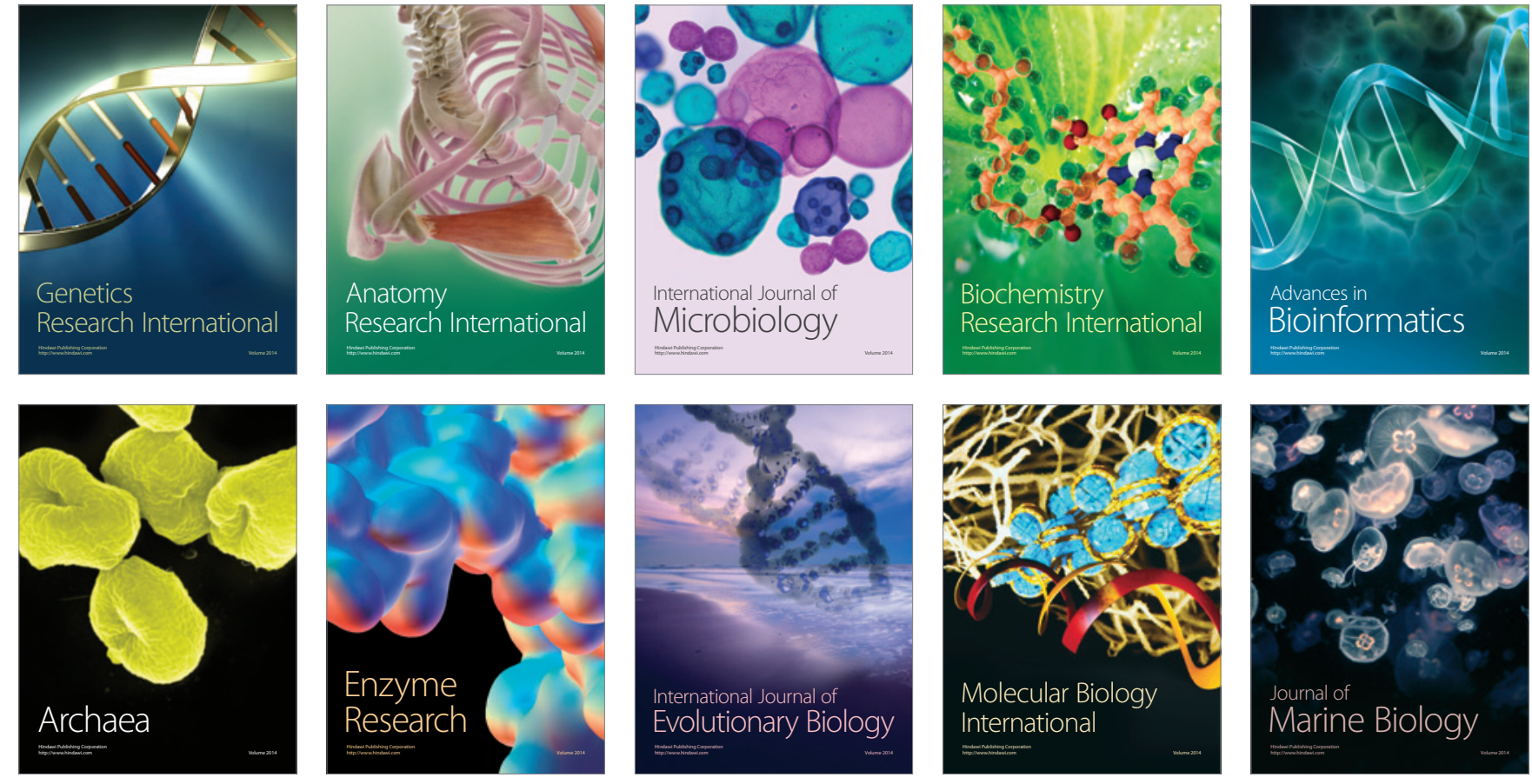